\title{
Quantum Entanglement Nature of Relativity of Angular Momentum and Inertia of Rotating Bodies
}

\author{
Boris Lagutin
}

Funding: The author(s) received no specific funding for this work.

Potential competing interests: The author(s) declared that no potential competing interests exist.

\section{Abstract}

In this experimental paper (preprint), the author analyzes how relativity of angular momentum and of inertia of rotating bodies may work physically (not mathematically). For example, all bodies, including astronauts, inside the International Space Station, have colossal moments of Inertia and colossal angular momentums which are physical properties of rotating bodies. These physical properties both depend on radii of rotation. All orbital trajectories have very large radii of rotation. However, the astronauts can easily move any bodies inside the International Space Station despite their colossal angular momentums and colossal moments of Inertia. The astronauts' capability to overcome the colossal inertial resistance of the bodies in the International Space Station is explained by relativity of angular momentum and of moment of Inertia. The relativity of the momentum and of the inertia of the bodies inside the International Space Station is just a statement. However, it is unclear how such relativity may work physically. Namely, it is not explained what exactly physically happens with the astronauts' bodies and other bodies so that the astronauts can easily move themselves and other bodies inside the International Space Station. An experiment described in this paper is aimed to understand what happens with not only astronauts' bodies but also other bodies which rotate relatively to each other. Notice that making mathematical manipulations like canceling out variables, for example a variable symbolizing a rotational radius, in physical equations does not remove such physical properties as angular momentum and moment of Inertia.

Introduction

Let's look at an astronaut who plays with a gyroscope inside the International Space Station in this video.

It is well seen that the astronaut easily rotates the gyroscope when the gyroscope does not spin. Notice that the flywheel of the gyroscope (not the whole gyroscope) spins. However, the spinning gyroscope flies away when the astronaut tries to rotate it. In other words, the astronaut must apply some significant force to change the direction of the spin axis of the spinning gyroscope, see Figure 1. 

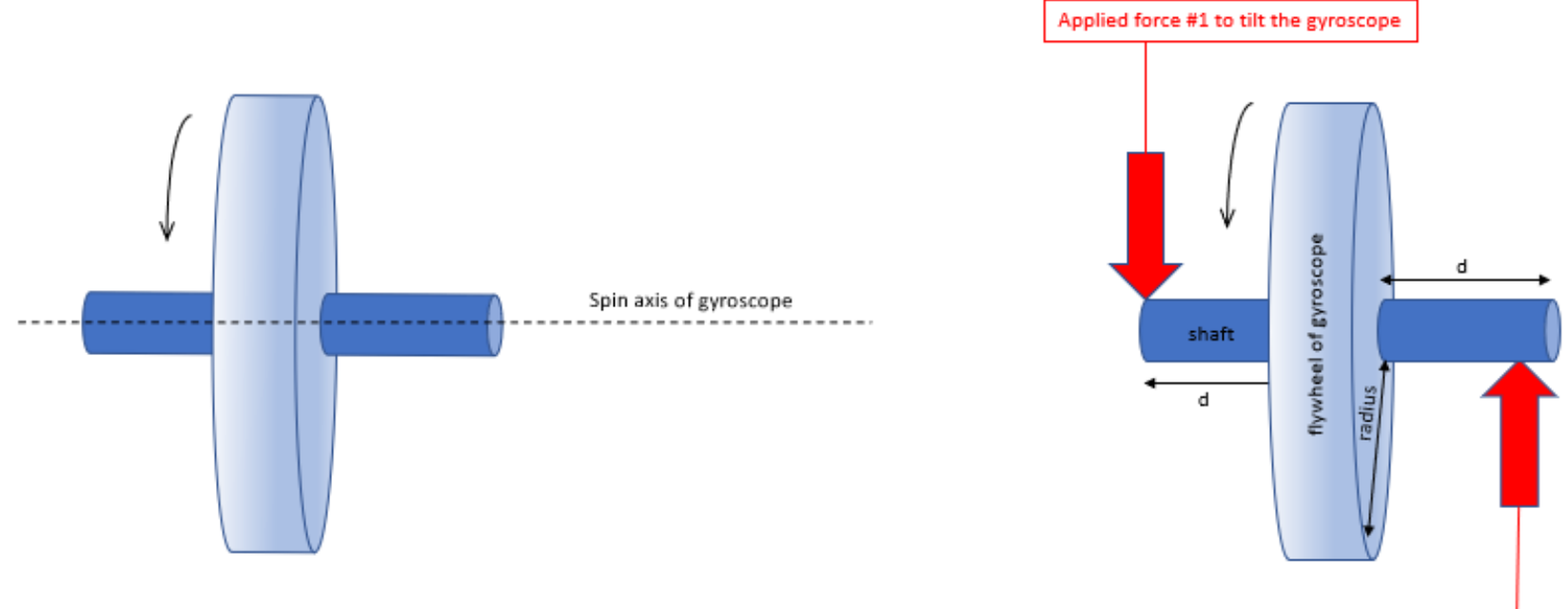

Applied force \#2 to tilt the gyroscope

Moreover, the gyroscope is inside International Space Station. Therefore, the whole gyroscope is constantly moving at the station orbital trajectory which is elliptical (almost circular). It means that even the nonspinning gyroscope (now as a point mass) has a colossal moment of Inertia and colossal angular momentum based on the following formulas [1, 2]:

$$
\begin{aligned}
& \square \approx \square \square^{2},(1) \\
& \square \approx \square \square,(2)
\end{aligned}
$$

where $\boldsymbol{I}$ is a moment of Inertia of the gyroscope, $\boldsymbol{m}$ is a mass of the gyroscope, $\boldsymbol{r}$ is a distance from a center of the gyroscope rotation, $\boldsymbol{L}$ is an angular momentum of the gyroscope, $\boldsymbol{w}$ is a angular speed of the gyroscope.

If assume that a mass of the gyroscope is nearly 0.2 kilogram ( 200 grams), then one may calculate a moment of Inertia of the whole gyroscope as a point mass rotating around the Earth at an approximately circular trajectory. The formula of the gyroscope moment of Inertia for elliptical trajectories contains a sine of an angle between the gyroscope position vector and the gyroscope velocity vector at each point [3]:

$$
\square=\square / \square=\square_{\square}^{2} \sin \left(\square_{\square}\right),
$$

where $\boldsymbol{r}$ symbolizes a distance between the gyroscope and its rotational center (magnitude of the gyroscope position vector) at each point of the gyroscope orbital trajectory, sin symbolizes a sine of an angle between the gyroscope position vector and the gyroscope velocity vector at each point of the orbital trajectory. Because the orbital trajectory of the International Space Station is almost circular; therefore, the moment of Inertia of the gyroscope at any point $p$ of the trajectory is:

$$
\square \approx \square \square_{\square}^{2}=0.2 \mathrm{~kg}(6,771,000.0)^{2} \square^{2}=9,169,288,200,000.0 \square \square \square^{2},
$$

and the gyroscope angular momentum if the International Space Station translational speed is 7.6 kilometers per second [4] or 0.0011312952296 radian per second is:

$$
\Pi \approx \Pi \Pi=9,169,288,200,000.0 \sqcap \Pi \Pi^{2} 0.0011312952296 \Pi \Pi \Pi / \Pi=10,373,172,000.0\left(\Pi \Pi \Pi^{2} \sqcap \Pi \Pi \Pi \Pi n\right) / \Pi .
$$


Hence, the following question raises: "How can the astronaut overcome the colossal moment of Inertia 9,169,288,200,000.0 kilograms per meters squared and angular momentum 10,373,172,000.0 kilograms per meters squared per radian per second of the gyroscope to move it so easily (see the video)?" It is obvious that the astronaut does not have such a terrific power in order to move even the non-spinning gyroscope at any direction. It is necessary to mention that the moment of Inertia and angular momentum are physical properties of a rotating body, in particular the gyroscope. These properties cannot be canceled out by any mathematical operations and manipulations. The fact is that the astronauts may move any bodies at any direction onboard the International Space Station. Some scientists may say that the astronaut(s) does not need to apply a terrific power because moment of Inertia and angular momentum are relative [5]. This statement is correct. But, saying this does not explain any physical nature how it may happen in the real world. What happens with a material (metal) of which the gyroscope is made with respect to the astronaut? What happens with the astronaut's body (muscles etc.) with respect to the gyroscope material? This situation looks like that the astronaut and the gyroscope are in one physical system where all physical properties and interactions are balanced so that we, observers including the astronauts, do not perceive any differences. Just for contrast, an astronaut, who is stationary with respect to the International Space Station, would need a terrific power to move the gyroscope at any direction.

An experiment described below models a situation, which is similar (but not the same) to the situation when the astronaut tries to rotate (change the spin plane of) the spinning gyroscope. However, a pusher is used instead of the astronaut's hand, see Figure 2.

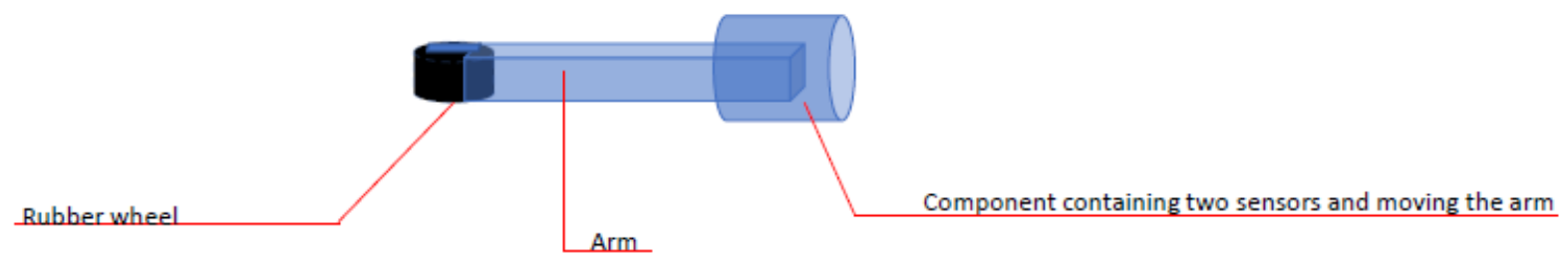

And a tested metal flywheel is used instead of the gyroscope, see Figure 3. 


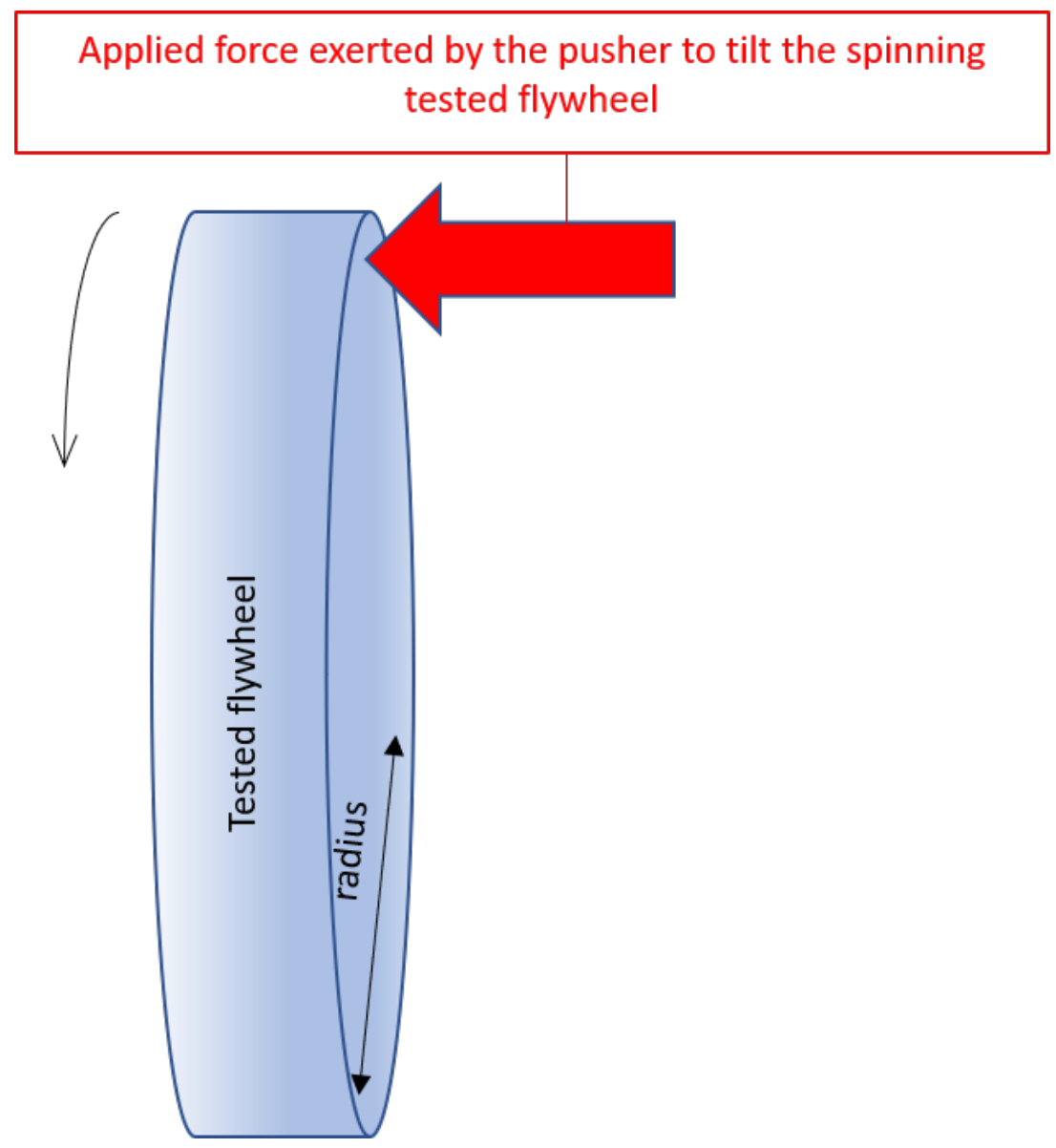

It is important to notice that the tested flywheel is assembled by using springs. In other words, the tested flywheel is not set to any central shaft, see Figure 4.

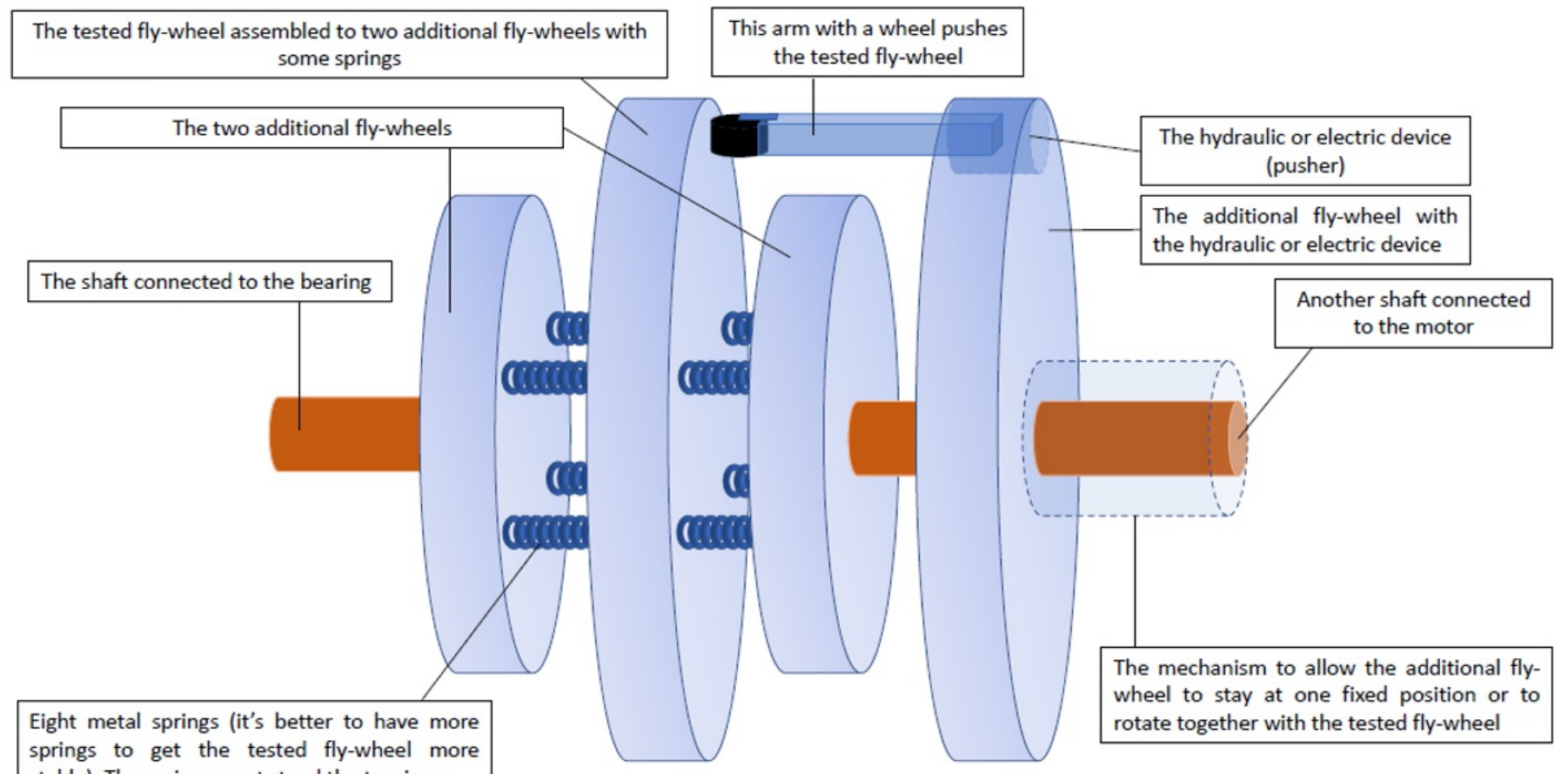

The pusher is fixed in the non-tested additional metal flywheel. The pusher does not spin by itself but may only rotate together with the additional flywheel. The latter is one of the additional flywheels. The whole 
set of the flywheels is connected to a programmable electromotor, see Figure 5.

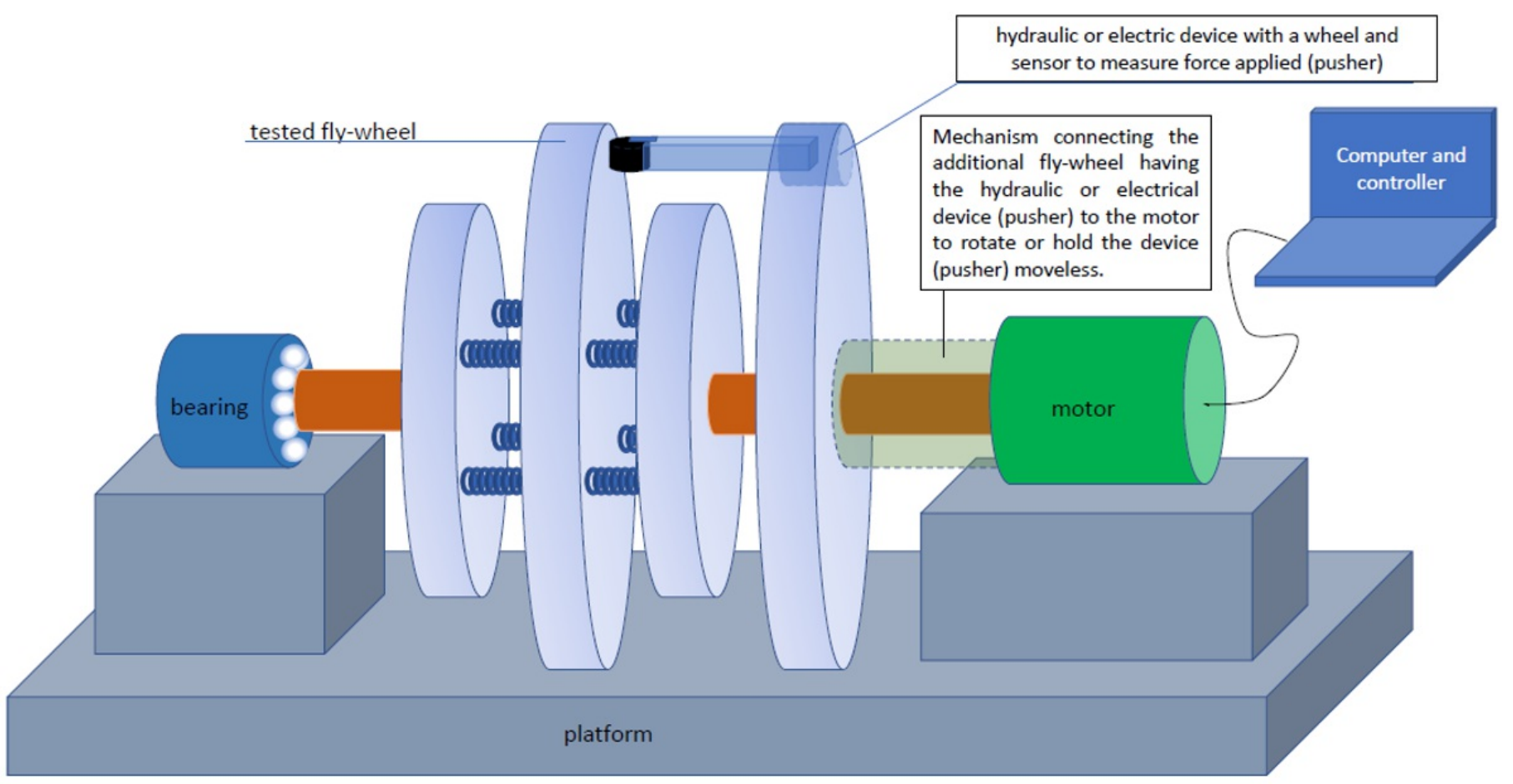

The pusher presses the tested flywheel perpendicularly to the surface of the additional flywheel in order to tilt the tested flywheel at the same angle $A$ in three situations, see Figure 6. 


\section{Situation \#1:}

\section{Situation \#2:}

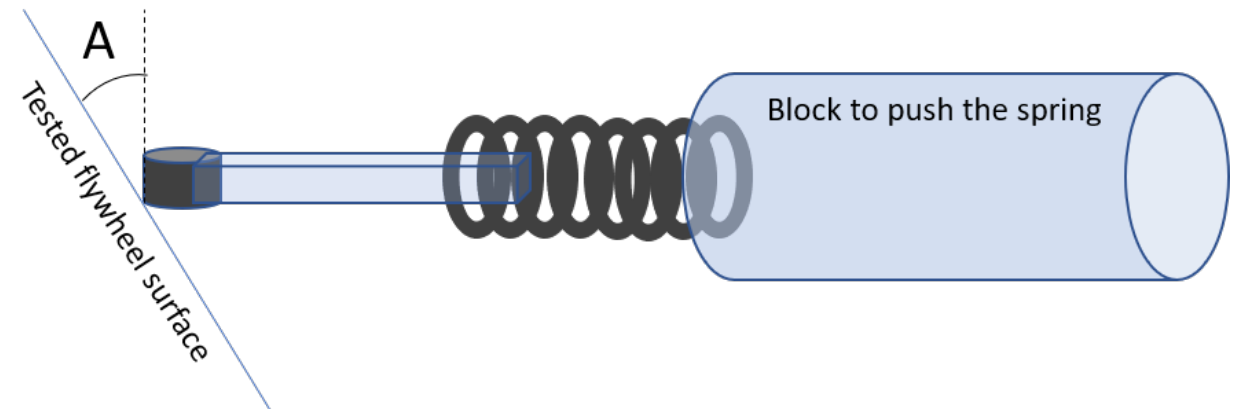

\section{Situation \#3:}
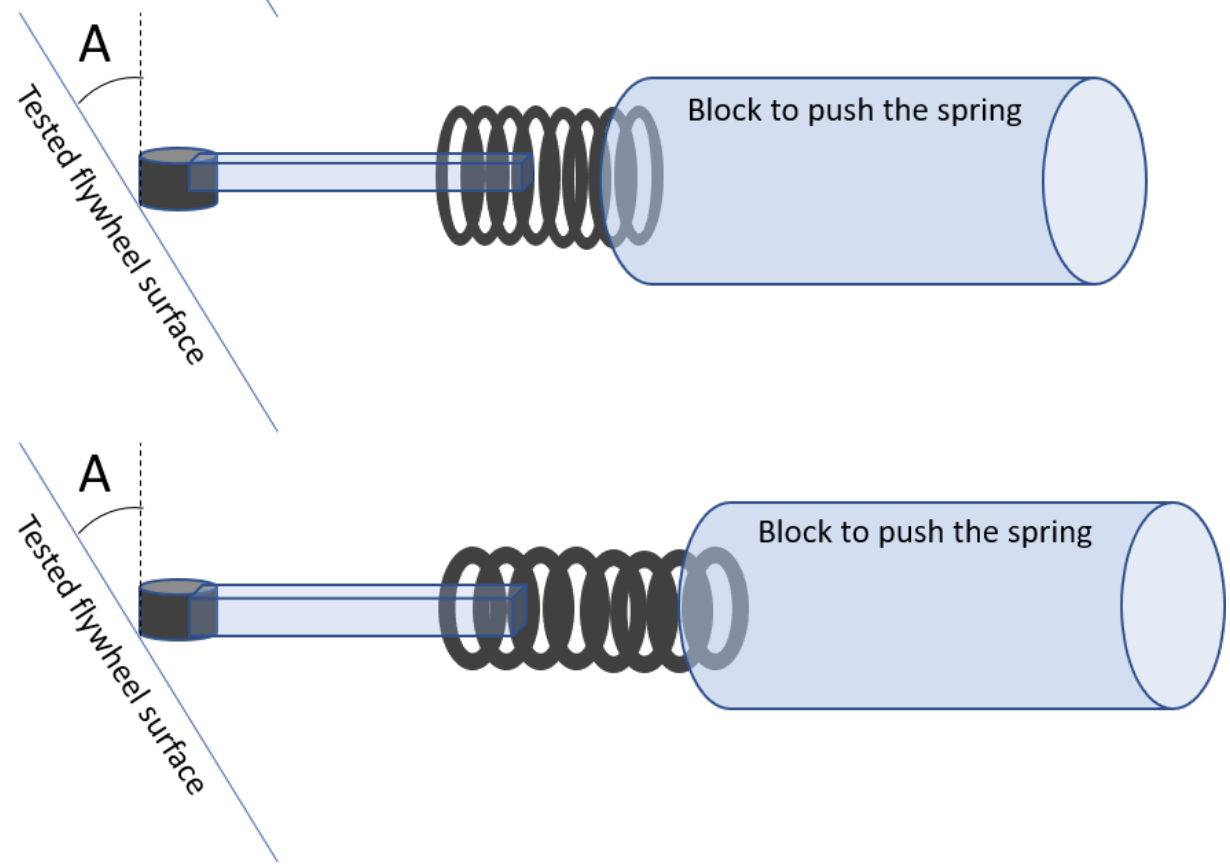

As a result, the pusher arm with the rubber wheel pass the same distance $\mathbf{D}$ in all the three situations, see Figure 7.

The initial position of the pusher arm with the rubber wheel (before pressing the tested flywheel):

The final position of the pusher arm with the rubber wheel (when it is pressing the tested flywheel):

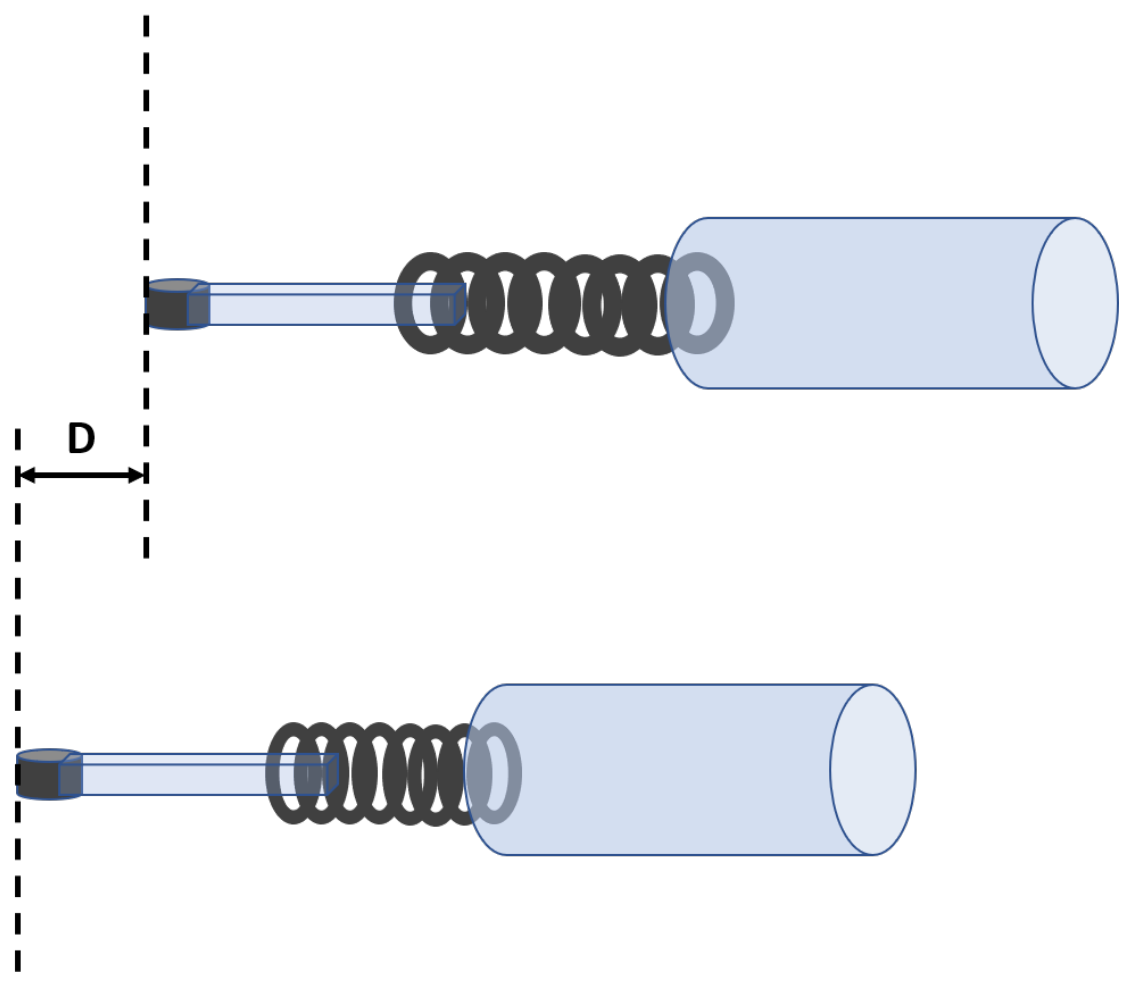


A force, which the pusher exerts on the tested flywheel to tilt the tested flywheel, is measured in the three situations:

1) when both the additional flywheel with the pusher and the tested flywheel do not spin at all (hereinafter named as Force \#1);

2) when the additional flywheel with the pusher is set at some fixed position but the tested flywheel spins at some chosen angular velocity (hereinafter named as Force \#2);

3) when the additional flywheel with the pusher and the tested flywheel spin all together at the same angular velocity (hereinafter named as Force \#3).

Let's choose one angular velocity (magnitude and direction) for the tested flywheel or the tested flywheel and the additional flywheel with the pusher for the second situation and the third situation appropriately. Notice that the first situation is just for measuring a force (Force \#1) which the pusher exerts on the tested flywheel at distinct sectors when the tested flywheel and the additional flywheel with the pusher do not spin, see Figure 8.

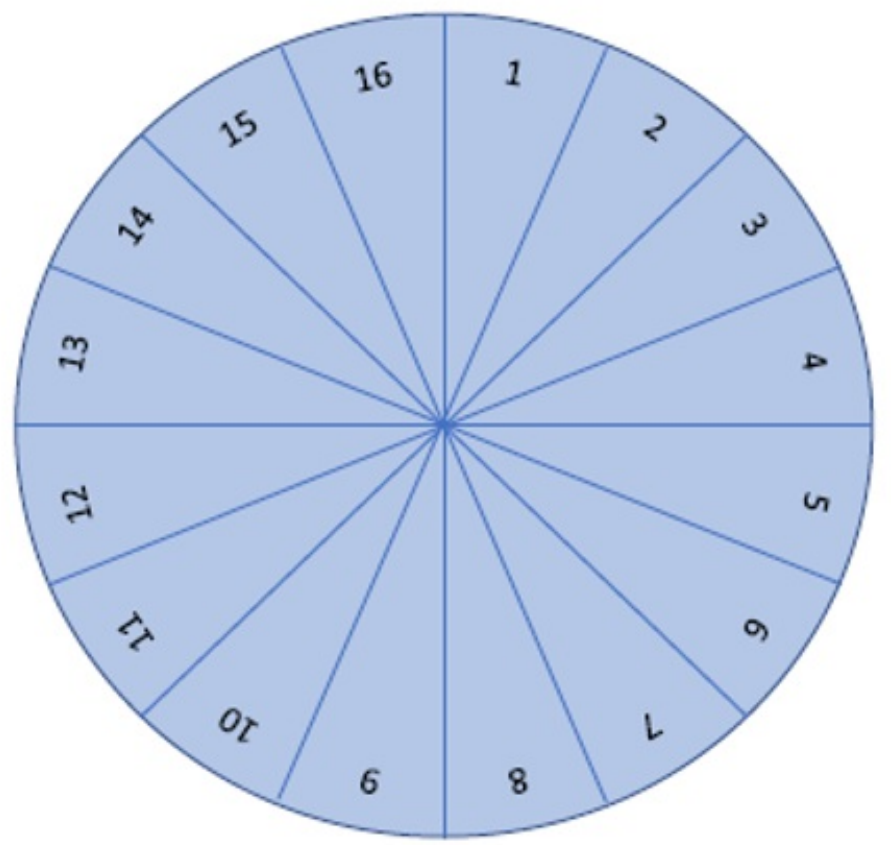

The number of the sectors depends on the tested flywheel radius: the more radius the more sectors

By measuring a force (Force \#1) applied for each sector in the first situation, one can further compare Force \#1 applied in the first situation with the forces (Force \#2 and Force \#3) applied in the other two situations. It is necessary to get the experiment clear results, so the flywheel resistance to a change of its spin plane caused by the flywheel angular momentum is only researched. The friction and tension forces caused by the springs and other components must be counted and excluded in the measurements of the force exerted by the pusher on the tested flywheel in the second situation and the third situation.

To exclude mistakes in measurements caused by centrifugal forces, the pusher is moved at the distance D while the additional flywheel with the pusher is rotating at a chosen angular velocity. It is executed 
without the tested flywheel in order to register a force which is needed to just move the pusher at the distance $\mathbf{D}$ while the pusher is experiencing forces caused by the rotation.

\section{Conclusion}

Based on the statement that angular momentum and moment of Inertia are relative, the following should be true. In the third situation the pusher should move (tilt) the spinning tested flywheel by exerting Force \#3 which is less than Force \#2 which the pusher exerts to tilt the spinning tested flywheel in the second situation. In other words, the angular momentum of and the moment of Inertia of the tested flywheel should not resist any change of the spin plane of the tested flywheel especially caused by the pusher in the third situation.

Based on the fact that the astronauts can easily move any bodies inside the International Space Station despite the colossal angular momentums of and the colossal moments of Inertia of the bodies, the author assumes that the pusher should exert Force \#3 which should be less than Force \#2 or equal to Force \#1.

If Force \#3 is less than Force \#2 or equal to Force \#1, the main experimental goal is understanding how physically it happens that the pusher can exert Force \#3 which is less than Force \#2. To reach the experimental goal, it is necessary to understand how the pusher works. It should be once again said that the tested flywheel must be tilted by the pusher at the same angle $\mathbf{A}$ for all the three situations; that is, the pusher arm with the rubber wheel is moved at the same distance $\mathbf{D}$. So if the spring in the pusher is less compressed while tilting the tested flywheel in the third situation than it is compressed in the second situation, then it is unclear what happens with the spring material in the third situation, see Figure 6 . The spring stiffness cannot change depending on the pusher angular speed (magnitude of the pusher velocity). Moreover, the angular momentum of the spinning tested flywheel cannot just disappear. Again, one may say that angular momentum is relative, but it is just a statement that does not explain how it works physically. Namely, what happens with materials of which the pusher and the tested flywheel are made relatively to each other.

The author assumes that the relativity of the angular momentum of and of the inertia of the tested flywheel may be based on quantum entanglement nature. The physical properties of bodies may be relative and anisotropic depending on their relative velocities (magnitudes and directions). However, a series of supplementary experiments is necessary to figure out the nature. In particular, various modifications of the pusher could be used for the experiment by measuring electricity supplied to the pusher in each situation.

If Force \#3 is equal to Force \#2, it means that the pusher overcomes the resistance caused by mostly the flywheel angular momentum. Then, it is absolutely unclear why the astronauts do not need to overcome the colossal angular momentums of and the colossal moments of Inertia of bodies inside the International Space Station. Force \#3 equal to Force \#2 compromises relativity of angular momentum and of moment of Inertia. 
Based on the said above, it seems that it is prospective to use a vector as a mathematical tool to describe the reality.[6] Though, there is a lot of work on this way in the author's view.

It is important to mention that the experiment is not related to the large moment of Inertia, so the moment of Inertia of the spinning tested flywheel is small and does not change if a mass of and a radius of the tested flywheel do not change. The experiment is much related to the angular momentum of the spinning tested flywheel because the angular momentum depends on the angular speed (magnitude of velocity) of the tested flywheel. The larger angular speed of the tested flywheel the larger angular momentum of the tested flywheel the harder one changes the plane of the spin of the tested flywheel.

References:

1. https://en.wikipedia.org/wiki/Moment_of inertia

2. https://en.wikipedia.org/wiki/Angular momentum

3. 11. Mass Moment of Inertia of Rigid Bodies - YouTube

4. https://en.wikipedia.org/wiki/International_Space_Station

5. https://en.wikipedia.org/wiki/Momentum

6. Carroll M. Sean (2021) Reality as a Vector in Hilbert Space, www.arXiv.org, Cornell University, March 2021: https://arxiv.org/abs/2103.09780 